\title{
Reversible suppression of Coulomb staircase in InP nanowires with light illumination
}

\author{
Hidenori Yamada $^{* a)}$, Toshishige Yamada ${ }^{\text {b),c) }}$, Andrew J. Lohn ${ }^{\text {c),d) }}$, and Nobuhiko P. Kobayashic),d) \\ ${ }^{\text {a) }}$ Department of Electrical and Computer Engineering, UC San Diego, La Jolla, California 92093 \\ ${ }^{b)}$ Center for Nanostructures, School of Engineering, Santa Clara University, Santa Clara, California 95053 \\ c) Department of Electrical Engineering, Baskin School of Engineering, UC Santa Cruz, Santa Cruz, California 95064 \\ ${ }^{d)}$ Nanostructured Energy Conversion Technology and Research (NECTAR), Advanced Studies Laboratories, UC Santa Cruz \\ and NASA Ames Research Center, Moffett Field, California 94035
}

\begin{abstract}
Detailed electron transport analysis is performed for an ensemble of conical indium phosphide nanowires bridging two hydrogenated $\mathrm{n}^{+}$-silicon electrodes. The current-voltage $(I-V)$ characteristics exhibit a staircase in dark with a period of $\sim 1 \mathrm{~V}$ at room temperature. The staircase is found to disappear under light illumination. This observation can be explained by assuming the presence of a tiny island within contributing nanowires. Electrons tunnel in and out of the island, resulting in the Coulomb staircase $I-V$. Applying light illumination raises the electron quasi-Fermi level and the tunneling barriers are buried, causing the Coulomb staircase to disappear.
\end{abstract}

Keywords - InP nanowire, Coulomb staircase, illumination.

\section{INTRODUCTION}

Indium phosphide nanowires (InP NWs) are becoming a popular research topic. Kobayashi et al. reported InP NW synthesis and electrical transport measurements on a simple photoconductor under light illumination [1]. NWs were grown from a pair of $n^{+}-\mathrm{Si}: \mathrm{H}$ electrodes facing each other, resulting in pairs of fused nanowires that make electrical paths between the electrodes. In this paper we have performed detailed analysis of the DC electron transport characteristics for the InP NW photoconductor devices in darkness and under light illumination by laser light (633 $\mathrm{nm}, 1.95 \mathrm{eV}$ ) at various optical power levels up to $5 \mu \mathrm{W}$. In the NW photoconductor devices, one electrode is used as a source and the other as a drain. Note that there is nothing that can act as a gate electrode. The light energy is significantly greater than the InP direct band gap $E_{\mathrm{g}}$ of 1.34 $\mathrm{eV}$, so appreciable electron-hole pair generation is expected.

We have examined several devices and observed increasing conductance because of electron-hole generation from illumination power. Nearly half of those devices showed a smooth $I_{\mathrm{d}^{-}} V_{\mathrm{d}}$ curve in darkness with a slight diverging nonlinearity and the differential conductance between the electrodes of the NW photoconductor device; $R_{\mathrm{PC}}=d I_{\mathrm{d}} / d V_{\mathrm{d}}$ changed from $\sim 2 \mathrm{nS}$ at $V_{\mathrm{d}}=0$ to $\sim 5 \mathrm{nS}$ at $V_{\mathrm{d}}$ $=5 \mathrm{~V}$. This behavior is well understood; the details for such devices are discussed in figure 3 of [2]. However, the remaining devices had an unusual $I_{\mathrm{d}}-V_{\mathrm{d}}$ curve in darkness that gradually disappeared with light illumination. The present paper focuses on the behavior of the latter type of device.

\section{EXPERIMENTS AND MODELING}

The resulting $I_{\mathrm{d}}-V_{\mathrm{d}}$ plot is considered for various light illumination powers measured in $\mu \mathrm{W}$. In the dark, $I_{\mathrm{d}}$ does not increase smoothly but rather has discrete jumps at regular intervals of $V_{\mathrm{d}}$, giving the darkness $I_{\mathrm{d}} V_{\mathrm{d}}$ a distinct staircase shape. In order to extract the $V_{\text {d }}$ period, we have subtracted the linear component of $I_{\mathrm{d}}$ and applied the Fourier transform to the result. The result has its peak at $2 \pi / \Delta V=6.6 \mathrm{~V}^{-1}$ or $\Delta V=0.95 \mathrm{~V}$. Any light illumination (above $0.64 \mu \mathrm{W}$ ) erases the staircase and leaves the linear component, making the $I_{\mathrm{d}}-V_{\mathrm{d}}$ characteristics Ohmic. In addition, the change is reversible: a device that has been illuminated will display, once returned to darkness, exactly the same staircase $I_{\mathrm{d}}-V_{\mathrm{d}}$ as before.

The basic theoretical model used in this paper to explain the experimental $I_{\mathrm{d}}-V_{\mathrm{d}}$ staircase is hereby a Coulomb staircase [3]. The main feature is an island in a 1D crystal system closely surrounded by a pair of tunneling barriers. The tunneling probability is so low that the number of electron on the island is quantized. The island is so small that the Fermi level inside the island is raised drastically even if a single extra electron tunnels in. These conditions allow the $I_{\mathrm{d}}-V_{\mathrm{d}}$ staircase to exist in darkness. When $V_{\mathrm{d}}$ is small, the island can accommodate only 0 charge. Since the island cannot change the number of electrons, no $I_{\mathrm{d}}$ can flow. As $V_{\mathrm{d}}$ is increased, the island tends to be positively charged, and $q$ and 0 charges (where $q$ $>0$ is the electron charge) can be accommodated. Then unit $I_{\mathrm{d}}$ can flow corresponding to one electron tunneling. As $V_{\mathrm{d}}$ is increased further, $2 q, q$, and 0 charges can be accommodated, and double the unit $I_{\mathrm{d}}$ can flow corresponding to two electron tunneling. This is how the discrete staircase steps are formed.

\footnotetext{
*Email: hyamada@soe.ucsd.edu
} 
Since the Si:H electrodes were $n^{+}$-doped and InP was unintentionally $n$-doped $10^{14}-10^{16} \mathrm{~cm}^{-3}$ in various different synthesis methods [4], electrons dominantly contribute to electrical transport. If light illumination is applied, only the electron quasi-Fermi energy $E_{\mathrm{Fn}}$ is considered relevant. Then with a higher $E_{\mathrm{Fn}}$, the barrier heights are effectively decreased and a reduced or eliminated (with sufficient power) staircase is expected, which is consistent with the experimental data.

\section{RESULTS AND DISCUSSION}

The total resistance of a photoconductor device $\left(R_{\mathrm{PC}}\right)$; $R_{\mathrm{PC}}=R_{\text {bulk }}+R_{\mathrm{T}}$, where $R_{\text {bulk }}$ represents the NW bulk resistance and $R_{\mathrm{T}}$ is the tunneling resistance. While $R_{\mathrm{T}}$ varies significantly among different fused NW pairs because of its exponential dependence on the barrier width, $R_{\text {bulk }}$ is expected to be rather comparable. We have confirmed the measured device conductance is a linear function of optical power $(P)$. This suggests that $R_{\text {bulk }} \gg R_{\mathrm{T}}$. Also, the Coulomb staircase requires isolation of the island, i.e., $R_{\mathrm{T}}$ $>R_{\mathrm{Q}}=12.9 \mathrm{k} \Omega$ where $R_{\mathrm{Q}}$ is the quantum resistance. Thus, in dark when the clear staircase is observable, $\mathrm{R}_{\text {bulk }} \sim 10$ $\mathrm{M} \Omega \gg R_{\mathrm{T}} \gg R_{\mathrm{Q}}$ for the fused NW pair dominating the photoconductor device's characteristics. For the other nondominant fused NW pairs, $R_{\mathrm{T}}>$ (or $>>$ ) $R_{\text {bulk }}$ and the resultant $R_{\mathrm{PC}}$ is much higher so that practically no current flows in these parallel connections. Under light illumination when the staircase disappears, $R_{\mathrm{T}} \leq R_{\mathrm{Q}}$ and $R_{\mathrm{PC}} \sim R_{\text {bulk }}$ is reinforced for the dominant NW pair. In some photoconductor devices, the staircase was absent even in dark, and this is interpreted as the source and drain capacitors $C_{\mathrm{s}}$ and $C_{\mathrm{d}}$ are not small enough, or $R_{\mathrm{T}} \gg R_{\mathrm{Q}}$ is not satisfied.

These capacitors are significantly smaller than the typical NW radius of $\sim 0.1 \mu \mathrm{m}$ around the fused portion. Furthermore, because InP NWs are unintentionally doped semiconductors and their surfaces are largely depleted, it is not the physical dimension of the fused portion that accounts for the capacitance. We have experimentally confirmed that our unintentionally doped InP blanket films are $n$-type with $n \sim 10^{15} \mathrm{~cm}^{-3}$. This means that our significantly wide NWs can have $n \sim 10^{15} \mathrm{~cm}^{-3}$, but our narrow NWs could have up to $n \sim 10^{16} \mathrm{~cm}^{-3}$. The depletion region width $w_{\text {dep }}$ is estimated with the abrupt planar junction formula $\left(2 \varepsilon V_{\text {surf }} / q n\right)^{1 / 2}$. We estimate $w_{\text {dep }} \sim 0.3 \mu \mathrm{m}$ for $n=10^{15} \mathrm{~cm}^{-3}$, and $w_{\text {dep }} \sim 0.1 \mu \mathrm{m}$ for $n=10^{16} \mathrm{~cm}^{-3}$. The staircase scenario demands that the radius and $w_{\text {dep }}$ be comparable near the fused portion of cone-shaped NWs whose radius is $\sim 0.1 \mu \mathrm{m}$ and that there be an active conducting region near the NW central axis. NW devices are electrically conducting even though NWs are connected via fused portions of $\sim 0.1 \mu \mathrm{m}$ and $\sim 10 \mathrm{~nm}^{2} \times 0.5 \mathrm{~nm}$ rectangular prism gap capacitors with $\sim 0.1 \mathrm{aF}$ can exist, corresponding to the observed staircase period of $\sim 1 \mathrm{~V}$. This is related to the charging energy for the Coulomb island and is unrelated to the height of the barriers that surround it. As shown below, the barrier heights are estimated at about $25-85 \mathrm{meV}$ and are much smaller than the island charging energy.

Next, we will discuss how $E_{\mathrm{F} 0}$ in dark is modulated by $P$ in InP. When $n \sim 10^{14}-10^{15} \mathrm{~cm}^{-3}, E_{\mathrm{F} 0}$ is located at $1.115-1.175 \mathrm{eV}$ above the top of the valence band $E_{\mathrm{v}}\left(E_{\mathrm{g}}=\right.$ $1.34 \mathrm{eV}$ ). Using e-h effective masses of 0.08 and 0.623 , effective conduction-band and valence-band densities $N_{\mathrm{c}}$ and $N_{\mathrm{v}}$ are $5.7 \times 10^{17} \mathrm{~cm}^{-3}$ and $1.1 \times 10^{19} \mathrm{~cm}^{-3}$, respectively. The intrinsic carrier density $n_{\mathrm{i}}$ is $10^{7} \mathrm{~cm}^{-3} . P$ creates the same density of excess e-h pairs $\Delta n=\Delta p . E_{\mathrm{Fn}}$ are expressed as a function of $P$. There is no practical difference in $E_{\mathrm{Fn}}$ between $n_{0}=10^{14} \mathrm{~cm}^{-3}$ and $10^{15} \mathrm{~cm}^{-3}$ except for $P=0$. The electron mobility $\mu$ was not measured and unknown. $E_{\mathrm{Fn}}$ and $E_{\mathrm{Fp}}$ were calculated for representative values, $\mu=34.7$ $\mathrm{cm}^{2} /$ Vs (a theoretical lower limit for a NW with $n \sim 10^{15}$ $\mathrm{cm}^{-3}$ ), $1000 \mathrm{~cm}^{2} / \mathrm{Vs}$, and $3000 \mathrm{~cm}^{2} / \mathrm{Vs}$ (an upper limit for intrinsic bulk InP). When $P=1 \mu \mathrm{W}$, however, quasi-Fermi levels are fairly insensitive to $\mu$, and $E_{\mathrm{Fn}} \sim 1.2 \mathrm{eV}$ and $E_{\mathrm{Fp}} \sim$ $0.30 \mathrm{eV}$. With further increase in $P, E_{\mathrm{Fn}}$ increases and $E_{\mathrm{Fp}}$ decreases gradually. For $P=0-5 \mu \mathrm{W}, \Delta E_{\mathrm{Fn}}=25-85$ $\mathrm{meV}$. When the potential barriers are comparable to $\Delta E_{\mathrm{Fn}}$, the Coulomb staircase scenario should appear. Note that the barrier height $(25-85 \mathrm{meV})$ is related to the drain current magnitude, while the charging energy is related to the staircase period $(1 \mathrm{~V})$.

We add the disappearance of Coulomb staircase with increasing gate bias in Ref. 5. In their experiment, the gate voltage increased the number of carriers, which has essentially the same effect as the light illumination in our experiment. Because our present photoconductor devices have no gate electrode, confirmation of Coulomb oscillation seen in drain current vs. gate voltage characteristics is currently not available.

\section{References}

[1] Kobayashi N P, Logeeswaran V J, Islam M S, Li X, Straznicky J, Wang S Y, Williams R S and Chen Y 2007 Appl. Phys. Lett. 91, 113116.

[2] Sarkar A, Logeeswaran V J, Kobayashi N P, Straznicky J, Wang S Y, Williams R S and Islam M S 2007 Proc. of SPIE 6768, 277.

[3] Yamada T, Chap. 7, "Nanoelectronics Applications" in Carbon Nanotubes ed. by Meyyappan M (CRC, Boca, Raton, 2004) 163-194.

[4] Martin T, Stanley C R, Iliadis A, Whitehouse C R and Sykes D R 1985 Appl. Phys. Lett. 46, 994.

[5] Thelander C, Martensson T, Bjork M T, Ohlsson B J, Larsson M W, Wallenberg L R, and Samuelson L 2003 Appl. Phys. Lett. 83, 2052. 